\title{
AtGRIP蛋白在拟南芥根冠细胞高尔基体反面网络分泌 囊泡的定位
}

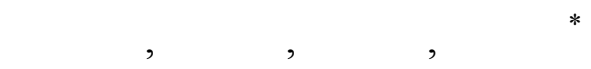

(1) 植物生理学与生物化学国家重点实验室, 中国农业大学生物学院, 北京 100094;

(2) 河北经贸大学生物科学与工程学院, 石家庄 050061

*联系人, E-mail: liyan@cau.edu.cn

摘要 动物与真菌细胞的 GRIP 蛋白定位于高尔基体反面网络, 并对高尔基体的结构与 功能有重要作用. 通过 RT-PCR 方法从拟南芥植株 RNA 中扩增得到 AtGRIP 的 cDNA; 利 用原核表达和亲和层析的方法, 获得 AtGRIP 部分片段的 GST 融合蛋白, 进而得到相应 蛋白的多克隆抗体. 利用纯化的 AtGRIP 抗体进行免疫印迹, 确认拟南芥 AtGRIP 蛋白的 分子量为 $92 \mathrm{kD}$, 并发现其在拟南芥根、茎、叶和花中均有表达; 免疫苂光标记 AtGRIP 和 Nag-GFP 的共定位说明, AtGRIP 蛋白分布于拟南芥细胞高尔基体; 免疫金标结合电子 关键词 GRIP 蛋白 高尔基体反面网络 高尔基体 根冠细胞 拟南芥 显微镜观察发现, AtGRIP 蛋白主要定位于拟南芥根冠细胞高尔基体反面网络的囊泡膜. 以上结果说明, 动植物细胞间 GRIP 蛋白在高尔基体上的定位是保守的; 同时也说明 AtGRIP 蛋白可能参与了对植物细胞高尔基体反面网络结构与功能的调控.

高尔基体是细胞内重要的细胞器, 其在细胞中 执行蛋白质修饰、加工和囊泡分类及分泌等功能. 高 尔基体反面的网状结构是位于高尔基体叠层反面的 一系列相互连接的管状结构. 它是一个高度动态且 复杂的膜结构网络, 主要功能是参与蛋白质的分类 与包装, 在糖蛋白和糖脂的加工和分泌过程中起重 要作用 ${ }^{[1,2]}$. 高尔基体反面的网状结构上存在许多结 合蛋白, 它们可能参与高尔基体反面的网状膜结构 的维持及囊泡分类及分泌功能的完成 ${ }^{[3,4]}$. 虽然植物 细胞高尔基体和动物细胞高尔基体在分布等方面有 一定的差异, 但近年来的研究发现, 植物细胞高尔基 体与动物细胞高尔基体具有许多相同的基本特征, 并可能执行类似的功能 ${ }^{[5]}$.

近年来, 在动物、真菌和原生生物细胞中发现一 种外周膜蛋白. 这类蛋白的 C末端含有一个大约 45 个氨基酸左右的保守序列, 该序列被称为GRIP结构 域, 有关蛋白被称为GRIP蛋白 ${ }^{[6 \sim 9]}$. 这些蛋白主要定
位在高尔基体上 ${ }^{[10]}$. GRIP蛋白分子内含有大量 $\alpha$-螺 旋, 能够形成棒状或纤维状结构. 进一步的研究表明, GRIP蛋白主要定位于动物和真菌细胞高尔基体反面 的网状结构上 ${ }^{[11]}$. 利用GFP融合技术将GRIP结构域 序列与 GFP基因融合转染动物或酵母细胞, 发现 GRIP结构域在其定位高尔基体的过程中起重要作用 [12]. GRIP蛋白在表达水平上的改变会导致高尔基体 反面网状结构的畸变, 并阻断膜运输途径 ${ }^{[13,14]}$. 这些 结果表明, GRIP蛋白在高尔基体反面网状结构的维 持和功能的完成方面起着重要的作用.

拟南芥中存在一个 GRIP结构域蛋白基因 (At5g66030), 被命名为AtGRIP. AtGRIP的cDNA长 $2367 \mathrm{bp}$, 编码 788 个氨基酸 ${ }^{[15]}$. 与动物、酵母序列相 同的是, AtGRIP氨基酸序列的C末端也含有一个由 42 个氨基酸组成的GRIP结构域; 该结构域与动物细胞 GCC185 和golgin-97 的GRIP结构域分别有 $50 \%$ 和 $47 \%$ 的相似性. 将AtGRIP的 GRIP结构域序列与 GFP 
基因融合, 转化烟草悬浮细胞, 结果发现其定位于高 尔基体上 ${ }^{[15]}$. 近来, 利用两种苂光蛋白转化方法, Latijnhouwers等人 ${ }^{[16]}$ 发现拟南芥AtGRIP蛋白定位于 烟草细胞高尔基体的反面，并发现序列中的卷曲螺 旋结构有助于C 端GRIP结构域与烟草细胞高尔基体 结合. 然而, 以上报道均是通过分析转化的烟草细胞 内拟南芥AtGRIP与苂光蛋白融合表达蛋白的分布, 判断AtGRIP在其中的可能定位; 而有关拟南芥细胞 内AtGRIP蛋白的实际分布情况，尚未见报道.

本研究将拟南芥 AtGRIP 蛋白在大肠杆菌内与 GST 融合表达, 纯化融合蛋白, 免疫动物制备抗体. 免疫印迹结果表明, AtGRIP 蛋白分子量为 $92 \mathrm{kD}$, 且 在拟南芥体内的表达没有组织特异性. 免疫苂光标 记结合共焦激光扫描显微镜观察确认, AtGRIP 蛋白 与高尔基体共分布. 免疫金标结合电子显微镜观察 发现, AtGRIP 蛋白定位于高尔基体反面网状结构的 分泌囊泡膜.

\section{1 材料和方法}

(i ) 植物材料. 所用拟南芥为 Columbia 型. 稳 定表达 Nag-GFP 的拟南芥纯合体植株由美国加州大 学戴维斯分校柳波教授惠赠.

(ii ) 拟南芥AtGRIP基因cDNA的克隆. 提取拟 南芥植株总RNA，参考GenBank提供的AtGRIP序列 信息(GenBank登录号: NM-180946), 通过 RT- PCR 扩增到拟南芥AtGRIP的cDNA全序列; 所使用引物为 AtGRIPSENSE

(5'-ATGTCCGAAGACAAGGAATCTGATG-3')和AtGRIPANTI (5'-CTATGAAAACGAGAATCTTGAGAAG-3'). 经测序确认, 得到的 AtGRIP cDNA序列与GenBank公布的序列一致.

(iii) AtGRIP 蛋白抗体制备与纯化. 利用 PCR 和酶切方法, 把 AtGRIP 基因片段(601 2262 bp)连接 到 pGEX-4T-1 原核表达载体上, 构建 pGEX-AtGRIP$\mathrm{C}^{\prime}$ 表达载体; 转化 E.coli BL21 (DE3) 菌株. 经 $1 \mathrm{mmol} / \mathrm{L} \mathrm{IPTG}$ 诱导, 在 $28^{\circ} \mathrm{C}$ 表达出可溶性的 GSTAtGRIP-C'融合蛋白. 用 Glutathione Sepharose 4B 从 超声破碎菌上清中纯化出 GST-AtGRIP-C'融合蛋白. 此纯化的 GST-AtGRIP-C'融合蛋白用来免疫兔子制 备多克隆抗体.

按Olmsted ${ }^{[17]}$ 报道的亲和纯化的方法, 利用 GST-AtGRIP-C'蛋白从抗血清中纯化特异的 AtGRIP 抗体. 纯化得到的 AtGRIP 抗体经 PBS 稀释、分装后
保存在 $-80^{\circ} \mathrm{C}$ 冰箱内.

(iv) SDS-聚丙烯酰胺凝胶电泳及免疫印迹. 拟 南芥植株或其根、茎、叶、花等器官经液氮研磨, 加 入提取缓冲液 $(5 \mathrm{mmol} / \mathrm{L}$ EDTA, $5 \mathrm{mmol} / \mathrm{L}$ EGTA, $1 \mathrm{mmol} / \mathrm{L}$ PMSF, $10 \mathrm{mmol} / \mathrm{L}$ DTT, $0.05 \% \mathrm{SDS}$, $20 \mathrm{mmol} / \mathrm{L}$ Tris-HCl，pH 8.0)提取相应蛋白. 参照 Laemmli ${ }^{[18]}$ 方法进行SDS-聚丙烯酰胺凝胶电泳.

免疫印记参照Towbin等人 ${ }^{[19]}$ 的方法, 一抗为经 纯化的AtGRIP多克隆抗体(工作浓度 1:1000), 二抗 为碱性磷酸酯酶交联的羊抗兔 IgG抗体(Sigma, 工作 浓度 $1: 60000)$. 显色液是Promega公司的碱性磷酸酯 酶试剂盒. 对照用免疫前血清代替一抗.

（v）免疫苂光标记及共聚焦激光扫描显微镜观 察. 植物材料的固定参照 $\mathrm{Li}$ 等人 ${ }^{[20]}$ 的方法; 拟南芥 根尖的压片参考Liu等人 ${ }^{[21]}$ 和Wasteneys等人 ${ }^{[22]}$ 的方 法. 免疫苂光标记参考Lee和Liu ${ }^{[23]}$ 报道的方法. 一抗 为经纯化的AtGRIP多克隆抗体(工作浓度 1:150), 二 抗分别为TRITC-交联的羊抗兔IgG (Sigma，工作浓 度 $1: 100$ )和FITC交联的羊抗兔 IgG (Sigma, 1:120). 对照用 3\%的BSA代替一抗. 制片在Zeiss LSM 510 META型共聚焦激光扫描显微镜下观察、拍照.

(vi) 免疫金标及电子显微镜观察. 超薄切片的 免疫金标参照van den Bosch等人 ${ }^{[24]}$ 及 Li等人 ${ }^{[25]}$ 的方 法. 一抗为经纯化的AtGRIP多克隆抗体 (工作浓度 $1: 150)$, 二抗为带有 $10 \mathrm{~nm}$ 胶体金颗粒的羊抗兔 $\mathrm{IgG}$ 抗体(Sigma, 1:60). 用JEM-100S电子显微镜进行观 察、拍照, 电子显微镜的电压为 $80 \mathrm{kV}$.

为确定 AtGRIP 的分布情况, 我们对金颗粒的分 布进行了统计分析. 我们统计了高尔基体、高尔基体 反面网络结构占细胞内总金颗粒中的比例; 共有 5 个 独立的细胞内金颗粒的分布被计算.

\section{2 结果}

2.1 拟南芥 AtGRIP 基因克隆、抗体制备和免疫印 迹分析

根据 NCBI 上报道的拟南芥 AtGRIP (At5g66030) 基因序列设计并合成引物，通过 RT-PCR 的方法从拟 南芥中克隆到 AtGRIP 的 CDS 序列. 测序结果表明, 我们克隆的 AtGRIP 序列与 NCBI 上报道的完全一致. 用原核表达得到的 AtGRIP 融合蛋白免疫兔子, 制备 出相应多克隆抗体.

拟南芥总蛋白经 SDS-PAGE 分离, 用纯化后的 
AtGRIP 抗体作为一抗, 对拟南芥总蛋白进行免疫印 迹分析, 结果在分子量大约 $92 \mathrm{kD}$ 处发生特异反应 (图 1(a), 3); 这表明 AtGRIP 的分子量约为 $92 \mathrm{kD}$; 此 分子量也与该蛋白理论计算的分子量相近. 用免疫 前血清对拟南芥进行印迹，未发现有任何条带出现 (图 1(a), 4). 这说明我们制备的抗体特异性较好.

拟南芥根、茎、叶、花等不同组织的总蛋白经 SDS-PAGE 分离后, 用 AtGRIP 抗体作为一抗, 进行 免疫印迹. 结果表明, AtGRIP 蛋白在拟南芥体内的表 达没有组织特异性(图 1(b)).

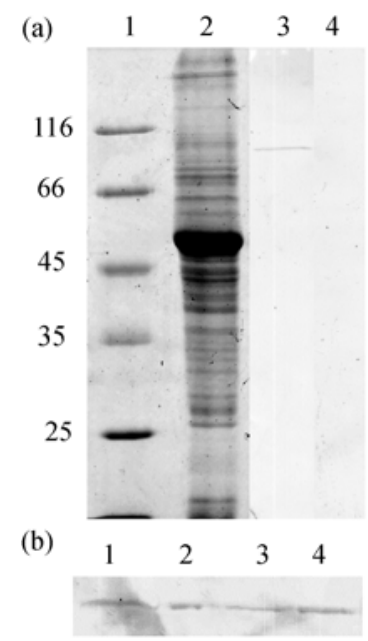

图 1 AtGRIP 蛋白的免疫印迹分析

(a) 拟南芥中总蛋白的 SDS-聚丙烯酰胺凝胶电泳 (2)和 AtGRIP 蛋 白的免疫印迹(3), 显示纯化的 AtGRIP 抗体特异地识别拟南芥总 蛋白中分子量约 $92 \mathrm{kD}$ 蛋白(3); 而免疫前血清代替一抗反应, 则 没有特异印迹带出现(4). 1 为标准分子量. (b) 拟南芥不同器官的 AtGRIP 蛋白免疫印迹, 表明该蛋白在拟南芥各器官中均有表达.

1 , 根; 2 , 茎; 3 , 叶; 4 , 花

\section{2 拟南芥 AtGRIP 的免疫苂光观察}

用纯化的 AtGRIP 抗体对拟南芥根尖细胞进行免 疫苂光单标记, 共聚焦激光扫描显微镜观察发现, AtGRIP 蛋白定位于拟南芥根尖细胞颗粒状细胞器 (图 2).

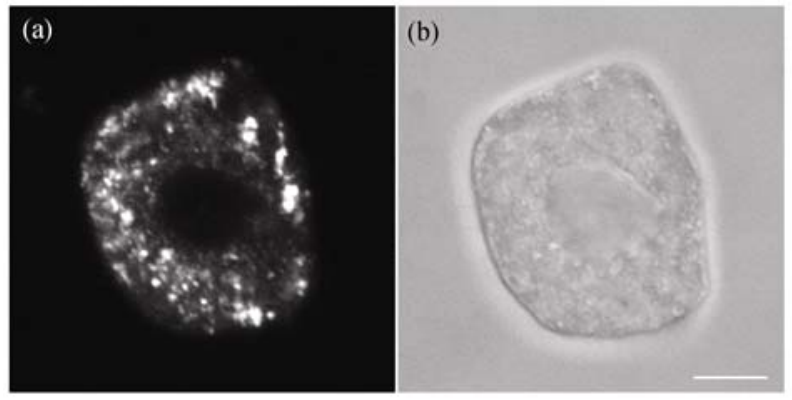

图 2 拟南芥根尖细胞内 AtGRIP 的免疫苂光定位及共聚 焦激光扫描显微镜观察

(a) AtGRIP 在拟南芥根尖细胞中分布在颗粒状细胞器上; (b) 同一 细胞微分干涉衬透射图. 标尺示 $5 \mu \mathrm{m}$

我们利用拟南芥Nag-GFP植株，用AtGRIP抗体 在表达Nag-GFP的拟南芥根尖细胞内进行免疫苂光 标记, 经共聚焦激光扫描显微镜观察发现, AtGRIP与 细胞内的GFP苂光存在部分共分布(图 3). 由于NagGFP特异地结合拟南芥细胞的高尔基体 ${ }^{[26]}$ ，因此可 确定，AtGRIP蛋白也定位于拟南芥根尖细胞的高尔 基体.

\section{3 拟南芥 AtGRIP 的免疫金标和电子显微镜观察}

基于绿色苂光蛋白转化和标记方法，人们已发 现过量表达的拟南芥AtGRIP-GFP融合蛋白定位于烟 草叶细胞高尔基体 ${ }^{[15,16,27,28]}$. 然而, 内源AtGRIP
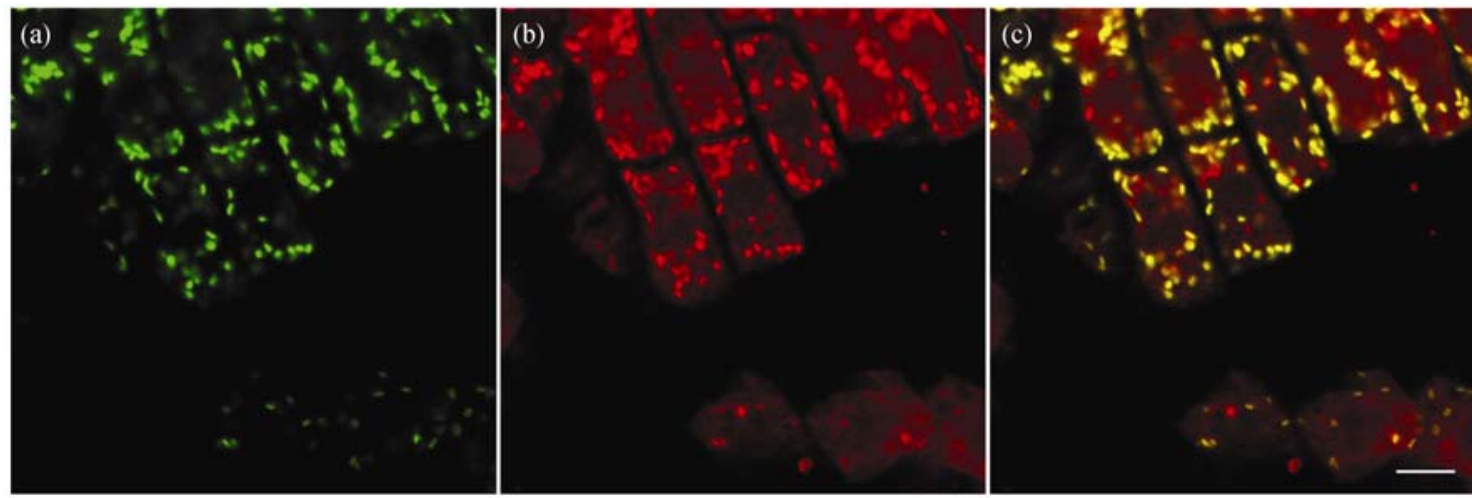

图 3 拟南芥根尖细胞内 AtGRIP 与 Nag-GFP 菼光双标记及共聚焦激光扫描显微镜观察

(a) Nag-GFP 显示的拟南芥根尖细胞中高尔基体的分布; (b) AtGRIP 蛋白在拟南芥根尖细胞中的免疫荧光定位; (c)为(a)和(b) 两图的叠加图, 显示拟南芥根尖细胞中 AtGRIP 蛋白与高尔基体部分共定位. 标尺示 $5 \mu \mathrm{m}$ 
在拟南芥细胞高尔基体上的分布情况尚不清楚. 为 此，我们用纯化后的AtGRIP抗体作为一抗对拟南芥 根冠细胞的超薄切片进行免疫胶体金标记, 并在电 子显微镜下进行了观察. 由于拟南芥根冠早期外周 细胞高尔基体的顺面和反面很容易通过其囊泡的大 小和形态进行分辨 ${ }^{[29,30]}$, 因此本研究显示的结果主 要来自对该类细胞的观察.

用纯化后的AtGRIP抗体进行标记, 在透射电子 显微镜下观察发现, 金颗粒多数分布在根冠细胞高 尔基体及其附近的囊泡上(图 4). 统计分析表明, 有 $73.4 \%$ 的金颗粒分布在高尔基体上. 进而，分析表明 有 $63.6 \%$ 的金颗粒分布于高尔基体具更多和更大的 囊泡一侧. 有关研究表明, 拟南芥根冠早期外周细胞 高尔基体反面往往有较大的分泌囊泡 ${ }^{[29]}$; 因此，根 据金颗粒主要分布于高尔基体具大囊泡侧, 我们可 以确认AtGRIP蛋白主要定位于高尔基体的反面. 进 而，我们发现金颗粒主要存在于在高尔基体反面分 泌囊泡膜的边缘(图 4, 箭头所示), 在高尔基体反面

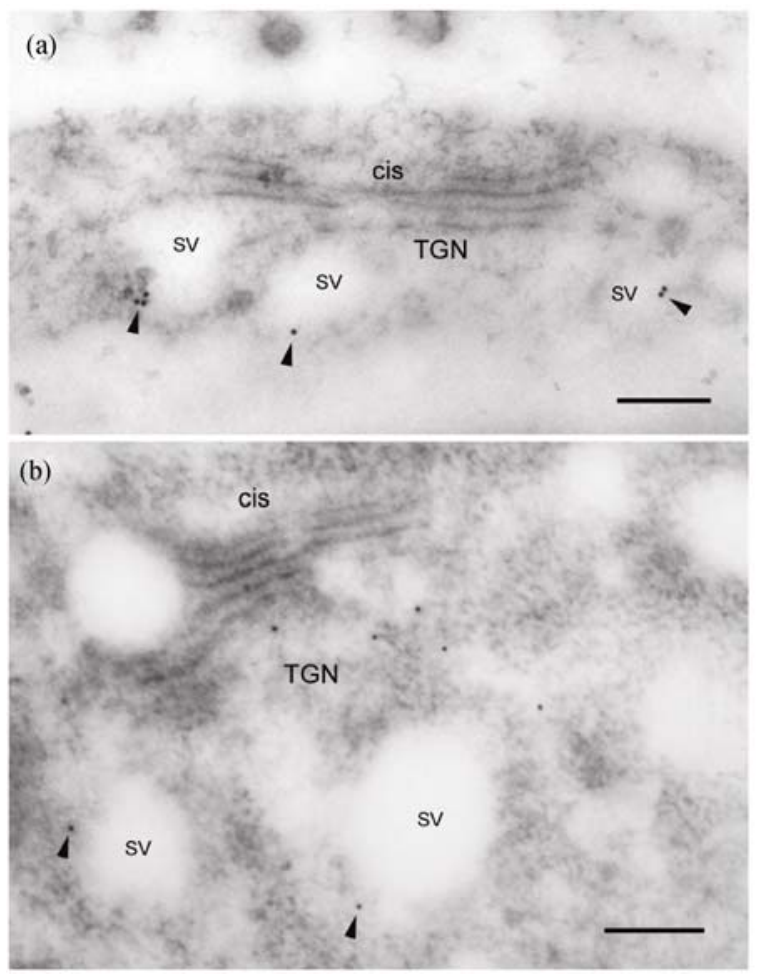

图 4 AtGRIP 蛋白在拟南芥根冠细胞中的免疫金标和透 射电子显微镜观察

显示经纯化的 AtGRIP 抗体标记, 电子显微镜观察确定 AtGRIP 蛋 白主要定位在高尔基体反面网络的囊泡膜上((a), (b)). SV, 分泌囊 泡; TGN, 高尔基体反面网络; cis, 高尔基体顺面. 标尺示 $200 \mathrm{~nm}$
膜囊上却很少. 以上结果说明, AtGRIP 蛋白主要定 位于拟南芥根冠细胞高尔基体反面网络的囊泡膜.

\section{3 讨论}

动物细胞的研究发现, GRIP蛋白具有一个功能 性的GRIP结构域, 是一种外周膜蛋白, 参与高尔基 体TGN结构的维持并调节囊泡的分泌与运输. 基因 组学研究表明, 拟南芥基因组中仅存在一个GRIP基 因 ${ }^{[15]}$. 本研究利用制备的AtGRIP多克隆抗体, 通过 免疫印迹方法, 发现拟南芥AtGRIP蛋白的分子量约 为 $92 \mathrm{kD}$; 此分子量与根据氨基酸数目理论计算的 AtGRIP分子量一致. 对拟南芥根、茎、叶和花进行免 疫印迹结果表明, AtGRIP在拟南芥植株内的表达并 没有组织特异性.

动物细胞GRIP蛋白定位在其高尔基体网络结构 上, 并且在细胞中参与高尔基体反面网络结构的维 持. 要研究植物细胞内 GRIP蛋白的功能, 了解其精 确的定位是非常重要的. 利用GFP转化技术, 人们已 发现拟南芥AtGRIP的GRIP结构域定位于烟草悬浮细 胞高尔基体 ${ }^{[15]}$. Latijnhouwers等人 ${ }^{[16,28]}$ 通过苂光双 标等方法, 确认拟南芥AtGRIP蛋白分布于烟草细胞 高尔基体反面. 然而, 目前的有关报道均是利用基因 转化方法, 通过GFP标记对外源的拟南芥AtGRIP融 合蛋白在烟草细胞中的分布进行研究; 但尚末见有 关内源AtGRIP蛋白在拟南芥中分布的报道，其在拟 南芥细胞高尔基体上的精确定位也有待确定. 另外, GFP融合蛋白转化与标记方法, 只能通过观察过量表 达的融合蛋白的分布, 对相关蛋白的实际定位进行 推测, 而无法直接观察到相关内源蛋白的实际存在 与分布; 而用特异抗体标记方法, 则可以直接确定细 胞相关内源蛋白的定位. 本研究利用AtGRIP多克隆 抗体对拟南芥根尖进行免疫苂光标记, 发现内源 AtGRIP蛋白确实定位于拟南芥根尖细胞高尔基体, 此结果印证了他人用GFP-AtGRIP转化方法在烟草细 胞中获得的结果. 此外, 虽然细胞内大多数AtGRIP 与Nag-GFP共定位, 但也发现细胞内少量AtGRIP的 标记是特异的, 未与Nag-GFP共定位, 推测这可能是 由于表达的Nag-GFP未与细胞内所有高尔基体结合 而造成的. 本研究利用免疫金标技术, 在电子显微镜 下观察发现, AtGRIP蛋白定位于拟南芥根冠细胞高 尔基体反面网络的囊泡膜. 目前, 已报道的动物细胞 GRIP蛋白主要是高尔基体的外周蛋白, 考虑到GRIP 
蛋白的保守性及AtGRIP蛋白序列中没有跨膜区，我 们推测AtGRIP可能是拟南芥细胞高尔基体反面网络 分泌囊泡膜上的外周蛋白.

在之前的有关报道中, 研究者发现GRIP蛋白除 定位于高尔基体反面网络的囊泡膜外，还分布于反 面高尔基体膜囊 228,31$]$. 然而, 我们发现AtGRIP蛋白 主要分布于拟南芥细胞高尔基体反面网络的囊泡膜, 而未定位于其反面高尔基体膜囊. 由于此前报道的 金标结果均是利用GFP抗体标记细胞内过量表达的 GFP-GRIP蛋白所获得, 因此, 我们推测有关GRIP蛋 白在细胞反面高尔基体膜囊上分布的结果有可能是
该蛋白过表达形成的假象. 内源的GRIP蛋白很可能 如我们用AtGRIP蛋白抗体标记的那样, 主要定位于 细胞高尔基体反面网络的囊泡膜，而不在反面高尔 基体膜囊上分布. 另一方面, 动物细胞GRIP蛋白的 过量表达会导致高尔基体反面网络结构的改变 ${ }^{[13]}$; 而抑制该蛋白表达则引起高尔基体分解为小的膜囊 叠层. 这些结果说明, GRIP蛋白参与了动物细胞 高 尔基体结构的维持 ${ }^{[14]}$. 而植物 GRIP蛋白定位于 高 尔基体反面网络分泌囊泡膜的结果，说明其可能参 与了对植物细胞高尔基体反面囊泡形成或分泌的调 控.

\section{参考文献}

1 Lippincott-Schwartz J, Cole N, Presley J. Unravelling Golgi membrane traffic with green fluorescent protein chimeras. Trends Cell Biol, 1998, 8: 16-20 [doi]

2 Lippincott-Schwartz J, Roberts T H, Hirschberg K. Secretory protein trafficking and organelle dynamics in living cells. Annu Rev Cell Dev Biol, 2000, 16: 557-589[doi]

3 Ladinsky M S, Wu C C, McIntosh S, et al. Structure of the Golgi and distribution of reporter molecules at 20 degrees C reveals the complexity of the exit compartments. Mol Biol Cell, 2002, 13: 2810-2825[doi]

4 Wylie F G, Lock J G, Jamriska L, et al. GAIP participates in budding of membrane carriers at the trans-Golgi network. Traffic, 2003, 4: $175-189$

5 Hawes C. Cell biology of the plant Golgi apparatus. New Phytol, 2005, 165: 29-44[doi]

6 Munro S, Nichols B. The GRIP domain-novel Golgi-targeting domain found in several coiled coil proteins. Curr Biol, 1999, 9: $377-380$ [doi]

7 Barr F A. A novel Rab6-interacting domain defines a family of Golgi-targeted coiled-coil proteins. Curr Biol, 1999, 9: 381-384[doi]

8 Kjer-Nielsen L, Teasdale R D, van Vliet C, et al. A novel Golgi-localisation domain shared by a class of coiled-coil peripheral membrane proteins. Curr Biol, 1999, 9: 385-388 [doi]

9 McConville M J, Ilgoutz S C, Teasdale R D, et al. Targeting of the GRIP domain to the trans-Golgi network is conserved from protists to animals. Eur J Cell Biol, 2002, 81: 485-495[doi]

10 Gillingham A K, Munro S. Long coiled-coil proteins and membrane traffic. Biochim Biophys Acta, 2003, 1641: 71-85[doi]

11 Derby M C, van Vliet C, Brown D, et al. Mammalian GRIP domain proteins differ in their membrane binding properties and are recruited to distinct domains of the TGN. J Cell Sci, 2004, 117: 5865-5874 [doi]

12 Barr F A, Short B. Golgins in the structure and dynamics of the Golgi apparatus. Curr Opin Cell Biol, 2003, 15: 405-413[doi]

13 Yoshino A, Bieler B M, Harper D C, et al. A role for GRIP domain proteins and/or their ligands in structure and function of the trans Golgi network. J Cell Sci, 2003, 116: 4441-4454 [doi]

14 Yoshino A, Setty S R G, Poynton C, et al. tGolgin-1 (p230, golgin-245) modulates Shiga-toxin transport to the Golgi and Golgi motility towards the microtubule-organizing centre. J Cell Sci, 2005, 118: 2279 - 2293[doi]

15 Gilson P R, Vergara C E, Kjer-Nielsen L, et al. Identification of a Golgi-localised GRIP domain protein from Arabidopsis thaliana. Planta, 2004, 219: 1050-1056[doi]

16 Latijnhouwers M, Hawes C, Carvalho C, et al. An Arabidopsis GRIP domain protein locates to the trans-Golgi and binds the small GTPase ARL1. Plant J, 2005, 44: 459-470[10i]

17 Olmsted J B. Affinitypurification of antibodies from diazotized paper blots of heterogeneous protein samples. J Biol Chem, 1981, 256: 11955-11957

18 Laemmli U K. Cleavage of structural proteins during the assembly of the head of bacteriophage $\mathrm{T}_{4}$. Nature, 1970, 227: 680-685[doi]

19 Towbin J, Sachelin T, Gordon J. Electrophoretic transfer of proteins from polyacrylamide gels to introcellulose sheets: Procedure and 
some application. Proc Natl Acad Sci USA, 1979, 76: 4350-4354 $\underline{\text { [doi] }}$

20 Li Y, Zee S Y, Liu Y M, et al. Circular F-actin bundles and a G-actin gradient in pollen and pollen tubes of Lilium davidii. Planta, 2001, 213: 722-730[doi]

21 Liu B, Marc J, Joshi H C, et al. A $\gamma$-tubulin-related protein associated with the microtubules arrays of higher plants in a cell cycle-dependent manner. J Cell Sci, 1993, 104: 1217-1228

22 Wasteneys G O, Willingale-Theune J, Menzel D. Freeze shattering: a simple and effective method for permeabilizing higher plant cell walls. J Microscopy, 1997, 188: 51-61 $\underline{\text { [doi] }}$

23 Lee Y R J, Liu B. Identification of a phragmoplast-associated kinesin related protein in higher plants. Curr Biol, 2000, 10: 797$800 \underline{\text { [doi] }}$

24 van den Bosch K A, Newcomb E H. Immunogold localization of nodule-specific uricase in developing soybean root nodules. Planta, 1986, 167: 425-436 [10i]

25 Li Y, Yen L F. Plant Golgi-associated vesicles contain a novel $\alpha$-actinin-like protein. Eur J Cell Biol, 2001, 80: 703-710[doi]

26 Lu L, Lee Y R J, Pan R, et al. An internal motor kinesin is associated with the Golgi apparatus and plays a role in trichome morphogenesis in Arabidopsis. Mol Biol Cell, 2005, 16: 811-823[doi]

27 Stefano G, Renna L, Hanton S L, et al. ARL1 plays a role in the binding of the GRIP domain of a peripheral matrix protein to the Golgi apparatus in plant cells. Plant Mol Biol, 2006, 61: 431-449 [doi]

28 Latijnhouwers M, Gillespie T, Boevink P, et al. Localization and domain characterization of Arabidopsis golgin candidates. J Exp Bot, 2007, 58: 4373-4386 [doi]

29 Staehelin L A, Giddings T H, Kiss J Z, et al. Macromolecular differentiation of Golgi stacks in root tips of Arabidopsis and Nicotiana seedlings as visualized in high pressure frozen and freeze-substituted samples. Protoplasma, 1990, 157: 75-91 [doi]

30 Dupree P, Sherrier D J. The plant Golgi apparatus. Biochim Biophys Acta, 1998, 1404: 259—270[doi]

31 Brown D L, Heimann K, Lock J, et al. The GRIP domain is a specific targeting sequence for a population of trans-Golgi network derived tubulo-vesicular carriers. Traffic, 2001, 2: 336-344 $\underline{\text { [doi] }}$

\section{《中国科学 C 辑: 生命科学》简介}

《中国科学 C 辑: 生命科学》(中文版) 和《Science in China Series C: Life Science》(英文版)是中国科学院主管、中 国科学院和国家自然科学基金委员会共同主办的自然科学综合性学术刊物, 主要刊载自然科学各领域基础研究和应用研 究方面具有创新性的、高水平的、有重要意义的研究成果, 由中国科学杂志社出版. 中、英文版是两个相对独立的刊物. 月 刊.

《中国科学 C 辑: 生命科学》(中文版) 被《中国科学引文数据库》、《中国学术期刊网》、《中国科学文献数据库》、 《中国数字化期刊》数据库收录, 《Science in China Series C: Life Sciences》(英文版)被 SCI, MEDLINE, CA, 俄罗斯文摘 杂志等数据库收录.

\section{栏目设置}

评述：综述所研究领域的代表性成果、研究进展，提出作者自己的见解以及对今后研究方向的建议.

论文: 报道化学各领域具重要意义的创新性科研成果.

快报：简明扼要地报道化学各领域最新研究成果的核心内容. 本栏目文章将优先发表.

\section{投稿方式}

投稿时使用在线投稿的方式，可访问中国科学杂志社网站 www.scichina.com

\section{联系方式}

地 址: 北京东黄城根北街 16 号

邮政编码: 100717

电话: (010) 64015399

传 真: (010) 64016350

电子信箱: life@scichina.org 